\title{
Etude de l'évolution morphologique de la Passe de l'Ouest (embouchure de la Gironde) - Projet Européen : SANDPIT
}

\author{
Claude GUILBAUD, Régis WALTHER, Florence GANDILHON \\ SOGREAH/LHF, \\ BP 172, 38042 GRENOBLE Cedex 9, France
}

\section{Résumé:}

Dans le cadre du projet européen SANDPIT, et en collaboration avec le Port Autonome de Bordeaux, nous nous sommes intéressés aux évolutions morphologiques à long terme du chenal de navigation (Passe de l'Ouest) et des bancs de sable situés à l'embouchure de la Gironde. Cette zone présente une morphologie (bancs de sable, chenal naturel de la Gironde, etc.) très variée, associée à des conditions hydrodynamiques (houles et courants) qui engendrent des mécanismes hydrosédimentaires de fortes amplitudes. Après analyse des données nature disponibles, un modèle d'évolution morphologique de l'embouchure a été construit. Il s’appuie sur des techniques mises au point au sein de SOGREAH et il est basé sur les logiciels du système TELEMAC (EDF/DRD).

\begin{abstract}
:
As part of the european project SANDPIT, and in collaboration with the "Port autonome de Bordeaux", we have studied the navigation channel's long term morphological evolutions ("Passe de l'Ouest") and the sand banks situated to the Gironde River's mouth. This area has a morphology (sand banks, Gironde river's natural channel,...) very diversified, combined with hydrodynamic conditions (waves and currents) which generate hydrosedimentary mechanisms with important variation. After an analysis of the available natural data, an evolution morphological model of the river mouth has been built. It relies on techniques developed in SOGREAH and it is based on TELEMAC's software (EDF/DRD).
\end{abstract}

Mots-clés: Evolution morphologique, Modélisation, Télémac.

\section{Le projet SANDPIT}

L'extraction massive de sable au niveau de l'avant-côte, c'est-à-dire à des profondeurs de 10 à $30 \mathrm{~m}$, dans des puits/zones d'emprunt et d'extraction minière de grande envergure sera nécessaire à l'avenir dans de nombreux pays d'Europe, afin de recharger les plages et les dunes littorales face à l'érosion littorale croissante (qui est due, par exemple, à l'augmentation prévue du niveau de la mer) mais aussi pour les besoins en matériaux de construction. Le principal objectif du projet SANDPIT (Une description complète du projet, ainsi que la liste des intervenants, est disponible sur le site Internet

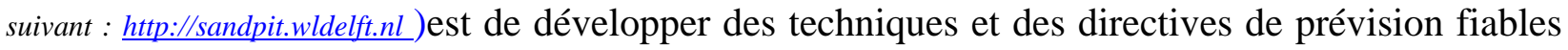
pour mieux comprendre, simuler et prévoir le comportement morphologique de ces zones d'extraction de sable de grande envergure, et les phénomènes de transport de sable au niveau de l'avant-côte et de la zone littorale voisine. 
Le projet s'articule autour de trois axes :

-Directives pratiques et outils de gestion relatifs à l'extraction minière sous-marine, -Collecte et analyse des données sur l'hydrodynamique, le transport de sable et la morphologie de l'avant-côte,

•Développement et perfectionnement des outils de prévision.

Les utilisateurs finaux du projet seront les suivants :

-autorités responsables de la gestion du littoral,

-consultants et entreprises de dragage,

•modélisateurs.

Le groupe de projet est constitué de 2 autorités responsables de la gestion du littoral, 5 instituts de recherche et 10 universités en provenance de 7 pays différents.

\section{Problématique}

L'entrée du chenal d'accès au Port de Bordeaux, dite «Passe de l'Ouest », est localisée à l'embouchure de l'estuaire de la Gironde. Le chenal utilise les passes naturelles entre le Banc de Montrevel au sud et le banc de la Coubre au nord (cf. figure 1). Dans son débouché, il est maintenu par un dragage d'entretien régulier car il passe à travers le banc du Matelier.

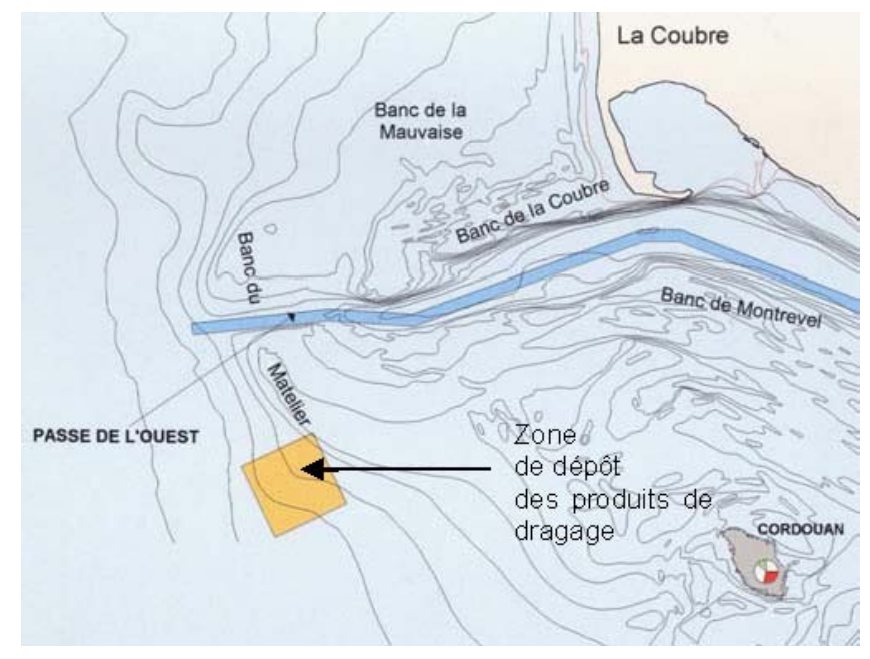

Figure 1 : Situation du chenal d'entrée du port de Bordeaux

Le chenal de navigation est soumis à des évolutions morphologiques importantes : le banc de la Coubre migre vers le sud et le banc du Matelier vers le nord. Au cours du $20{ }^{\text {èe }}$ siècle, du fait de la migration, associée à une forte sinuosité, de ces deux bancs, le Port de Bordeaux a été conduit par deux fois à créer un nouveau chenal : en 1930 et en 1980. En association avec le Port de Bordeaux, nous nous sommes intéressés aux évolutions morphologiques à long terme du chenal de navigation (Passe de l'Ouest) et des bancs de sable situés à l'embouchure de la Gironde. Les objectifs de cette étude sont :

- de développer un outil capable de représenter les phénomènes hydrosédimentaires dans et autour de la Passe de l'Ouest, 
- d’appliquer le modèle aux évolutions bathymétriques moyennes à long terme ainsi que celle liées à des épisodes de tempêtes océaniques, l'Ouest.

- de compléter la compréhension des phénomènes hydro-sédimentaires autour de la Passe de

\section{Les données nature}

Le Port Autonome de Bordeaux nous a fourni les relevés bathymétriques annuels de la Passe de l'Ouest depuis 1980. L'ouverture du chenal actuel date de 1981, après le dragage de 14,6 $\mathrm{Mm}^{3}$. Jusqu'en 1997, le volume annuel moyen dragué dans le chenal était de l'ordre de $0.36 \mathrm{Mm}^{3}$ (soit 5 fois moins que dans l'ancien chenal).

Depuis 1997, le volume moyen annuel dragué est de l'ordre de $0.88 \mathrm{Mm}^{3}$. Cette augmentation est due à la fermeture en 19961997 de l'ancien chenal de navigation qui jouait le rôle de « fosse » et retenait les apports sableux sur la nouvelle Passe de l'Ouest. L'évolution moyenne du fond entre 1994 et 2000 est présentée sur la figure 2.

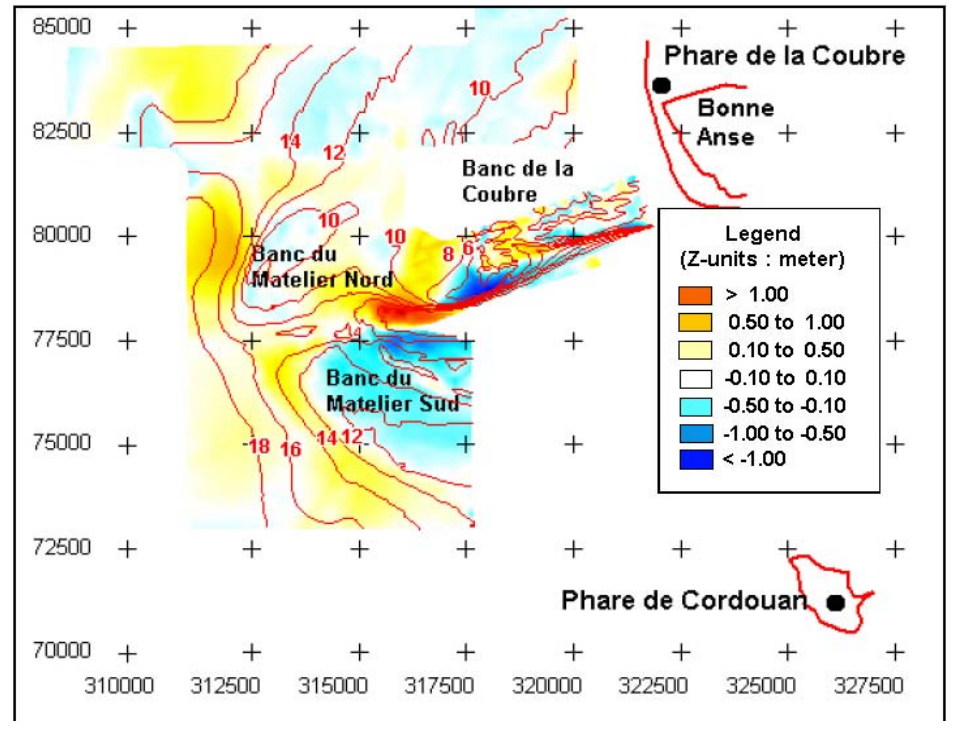

Figure 2: Evolution des fonds entre 1994 et 2000 ramenée à une année

Une analyse des évolutions morphologiques, entre 1981 et 2000, sur une zone précise (figure 3 , limites en bleu) montre que sa partie nord présente une tendance croissante au dépôt de sédiment alors que la partie sud présente une tendance stable à l'érosion (figure 4). Le bilan sur la totalité de la zone délimitée en bleu (figure 3) montre une croissance du dépôt. 


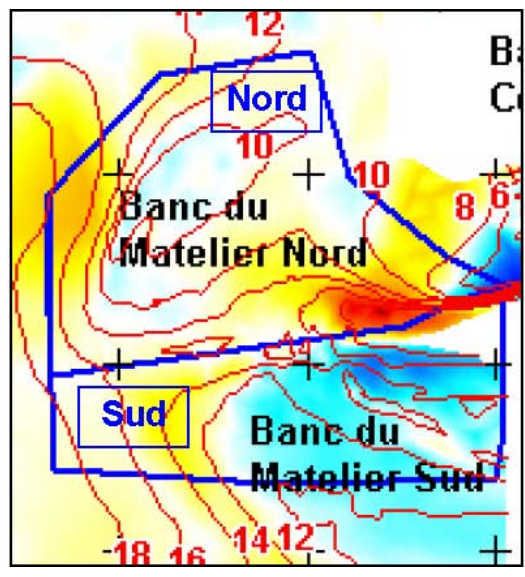

Figure 3: Délimitation des zones

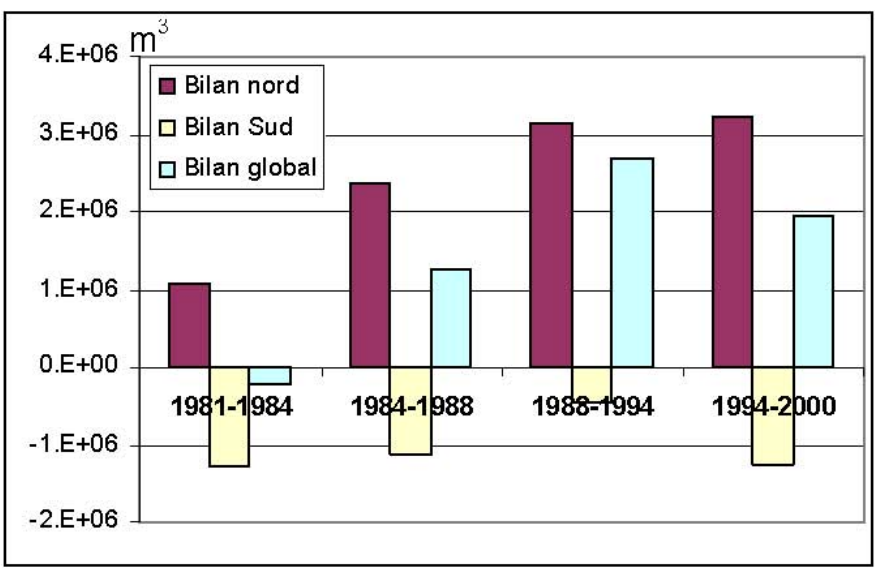

Figure 4: Histogramme des bilans d'évolutions morphologiques. des bilans morphologiques.

L’étude détaillée de la période 1994-2000 sur une étendue plus large, montre une tendance à l'érosion (en bleu) de la partie Est au profit de la partie Ouest (dépôt en rouge) de la zone de la Passe de l'Ouest (figure 5).

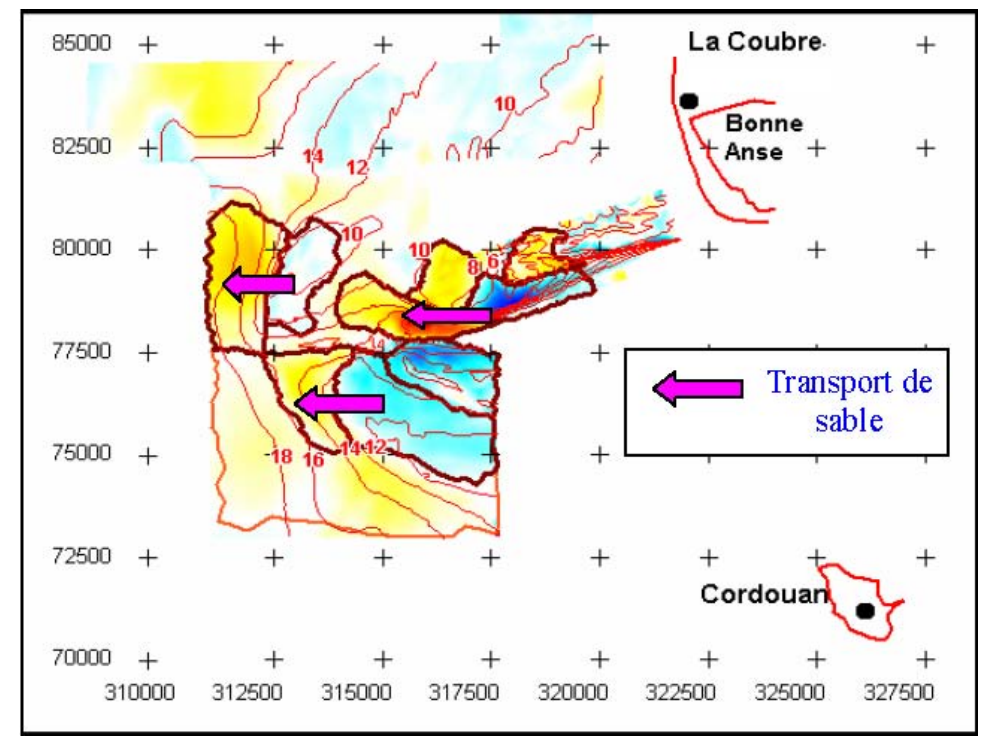

Figure 5 : Processus dominants de transports de sable

Les flèches roses indiquent les directions des transferts de sable. On observe une dominance des courants de jusant (transfert Est-Ouest) sur ce transport. 


\section{Stratégie de modélisation}

Afin de mieux comprendre les phénomènes mis en jeu dans cette zone, nous avons construit un modèle hydrosédimentaire de l'embouchure. Ce modèle s’appuie sur des techniques d'imbrication mises au point au sein de SOGREAH et basées sur les logiciels du système TELEMAC (EDF/DRD). Ce modèle s'appuie sur quatre niveaux de modélisation :

1-Un modèle hydrodynamique bidimensionnel de l'ensemble du golfe de Gascogne (TELEMAC-2D), qui fournit les conditions aux limites au modèle local (2).

2-Un modèle hydrodynamique bidimensionnel local de la façade aquitaine reproduisant les effets de « chasse » de la Gironde (TELEMAC-2D),

3-Un modèle de propagation de la houle de la façade aquitaine (TOMAWAC).

4-Un modèle morphologique de l'embouchure de la Gironde (SISYPHE), qui utilise les informations de courants (U et V) et de surface libre (SL) en provenance du modèle local (2) et de houles (Hs et Tp) en provenance du modèle de houle (3).

L'enchaînement de ces modèles est présenté sur la figure 6. Lorsque l'évolution du fond issue du logiciel Sisyphe est suffisante, on recalcule l'hydrodynamique et la houle pour la bathymètrie mise à jour.

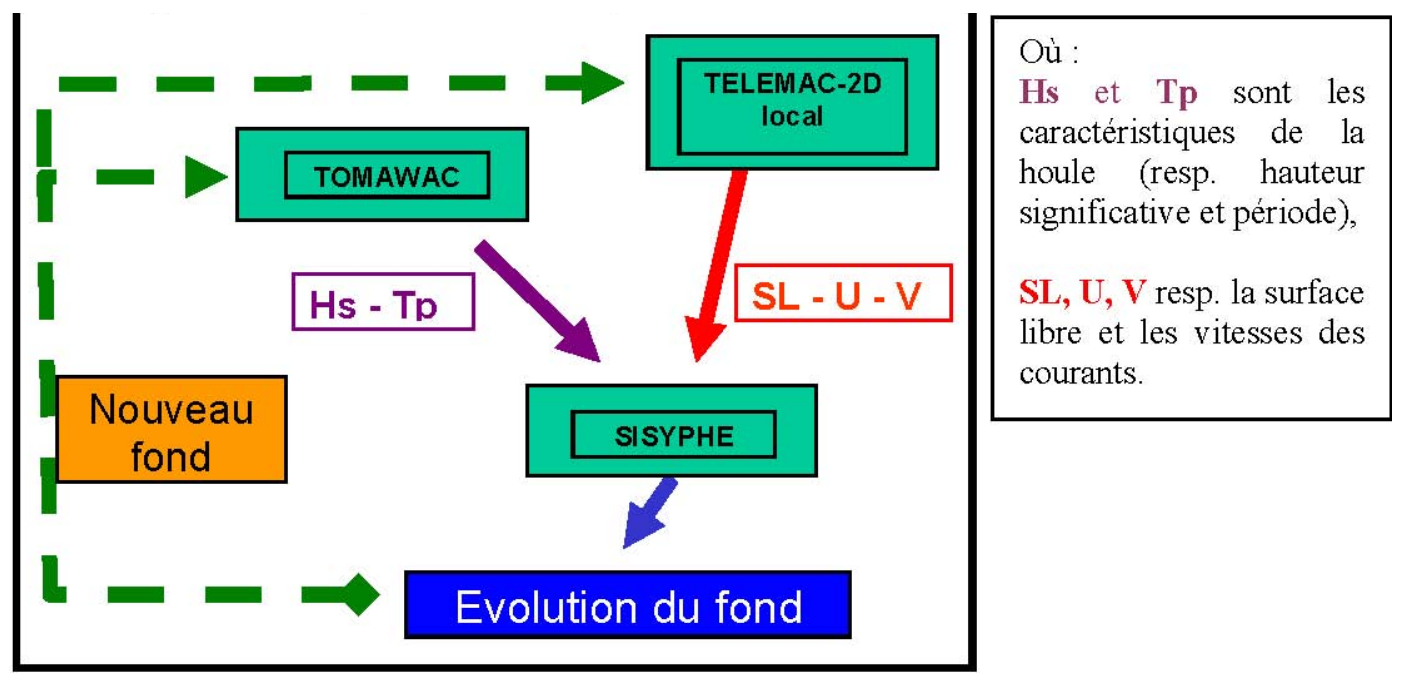

Figure 6 : Fonctionnement du modèle morphologique

Afin de reproduire les effets de la houle, des courants de marée et le débit de la Garonne et de la Dordogne, nous avons utilisé en première approche des conditions moyennes représentatives existant dans la zone étudiée :

-l’hydrodynamique est représentée par une marée de coefficient 90 qui correspond à la marée « morphologique »,

-les conditions de houle au large (conditions limites) sont les conditions représentatives du climat de houle sur la zone (Hs=1.0m et $\mathrm{Tp}=6.0 \mathrm{~s})$,

-le débit de la Garonne correspond au débit moyen annuel observé (QGaronne $=650 \mathrm{~m}^{3} / \mathrm{s}$ ) ; il en est de même pour le débit de la Dordogne (QDordogne=350m $/ \mathrm{s}$ ),

-les caractéristiques des sédiments utilisées dans les calculs sont celles observées sur le site $\left(\mathrm{d}_{50}=0.3 \mathrm{~mm}\right)$. 


\section{Les premiers résultats}

Nous avons concentré notre travail sur la reproduction des phénomènes morphologique sur une période assez longue (1994-2000). Cela permet de lisser l'impact des phénomènes hydrométéorologiques ponctuels. Nous présentons ici, quelques exemples des résultats obtenus par les modèles mis en œuvre. Les résultats du modèle hydrodynamique local montrent que les courants générés par la marée et la vidange de l'estuaire atteignent des intensités de l'ordre de $2.0 \mathrm{~m} / \mathrm{s}$ au jusant et à basse mer, ils sont contraints en direction par le chenal naturel de la Gironde.
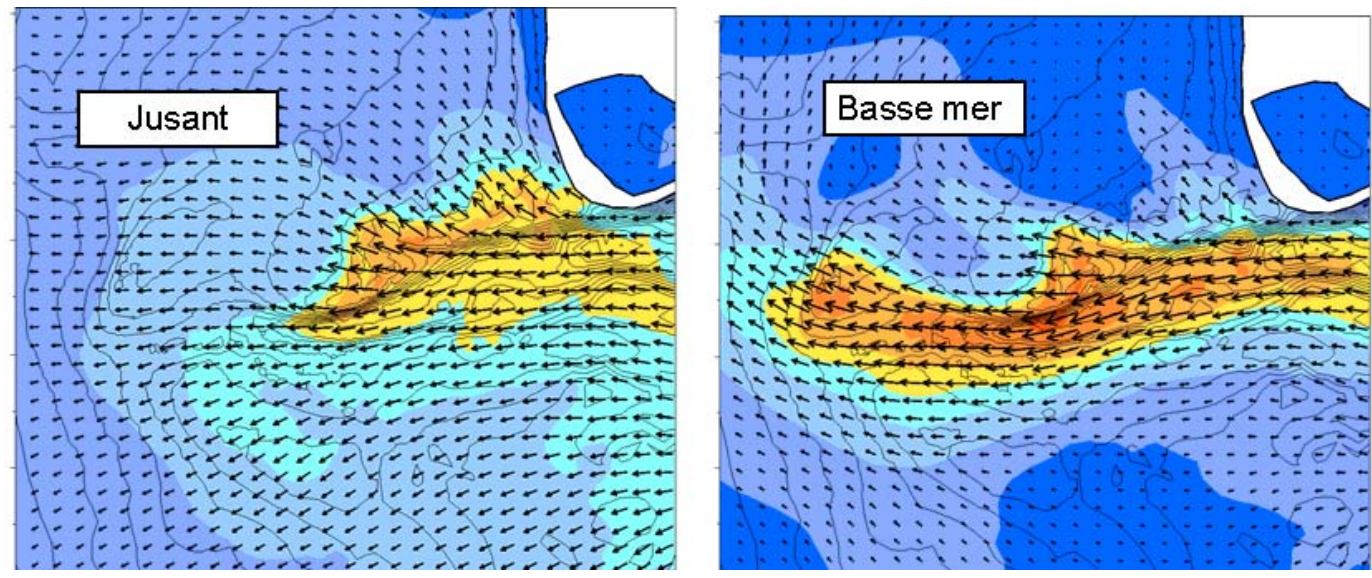

Figure 7 : Courants de jusant (gauche) et de basse mer (droite) à l'embouchure de la Gironde. La longueur des vecteurs et les couleurs donnent une indication de l'intensité.

Sur la double figure 7, on retrouve la dominance des courant de jusant et de marée basse comme le suggéraient les transferts de sable observés sur les données nature (figure 5). Les courants de flot et de haute mer ont des intensités inférieures à $1 \mathrm{~m} / \mathrm{s}$ (figure 8). Plus faible que pour la basse mer et le jusant, ils sont orientés nord-sud sur le banc de la Coubre et contribuent vraisemblablement à la dérive de celui-ci vers le sud.
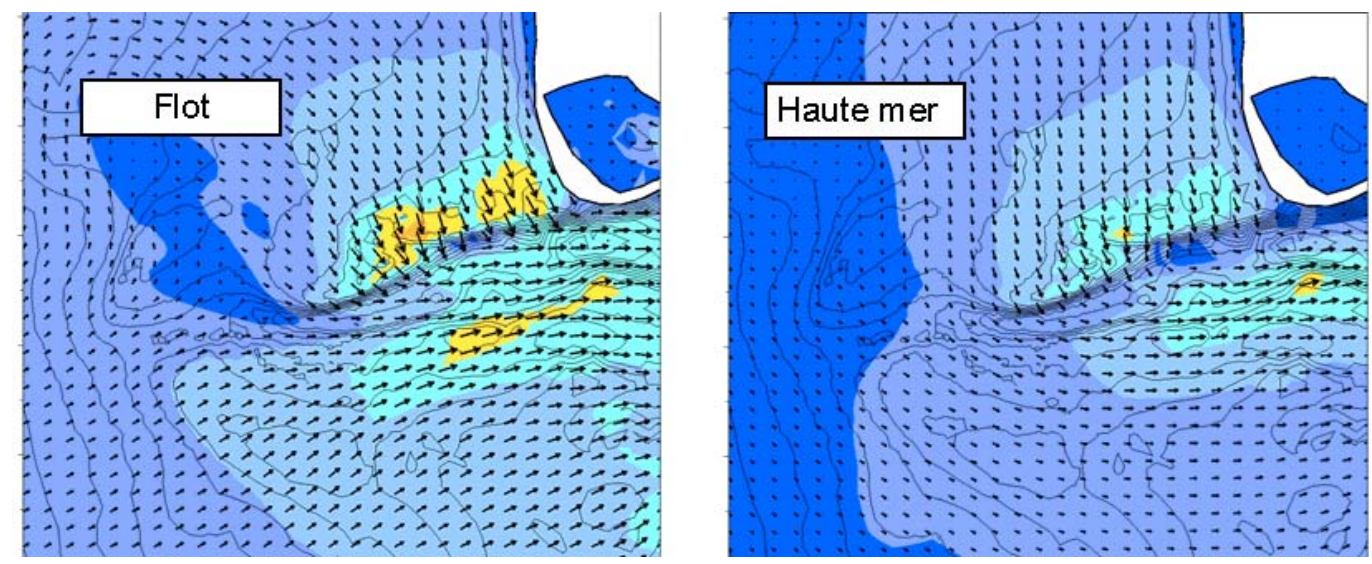

Figure 8 : Courants de flot (gauche) et de haute mer (droite) à l'embouchure de la Gironde. La longueur des vecteurs et les couleurs donnent une indication de l'intensité. 
La comparaison entre les mesures et les résultats du modèle, des évolutions morphologiques entre 1994 et 2000 ramenées à une année, est présentée sur les figures 9 et 10.

On retrouve qualitativement les mêmes répartitions pour les zones de dépôt et les zones d'érosion. Quantitativement, les valeurs des dépôts et des érosions sont surestimées par rapport à la réalité. Une analyse plus détaillée des données nature, basée sur des zones clé d’évolution (banc de la Coubre, déplacement du chenal de navigation vers le sud, évolution des deux parties du banc du Matelier), nous permettra d'effectuer un calage du « temps morphologique » à utiliser pour le modèle.

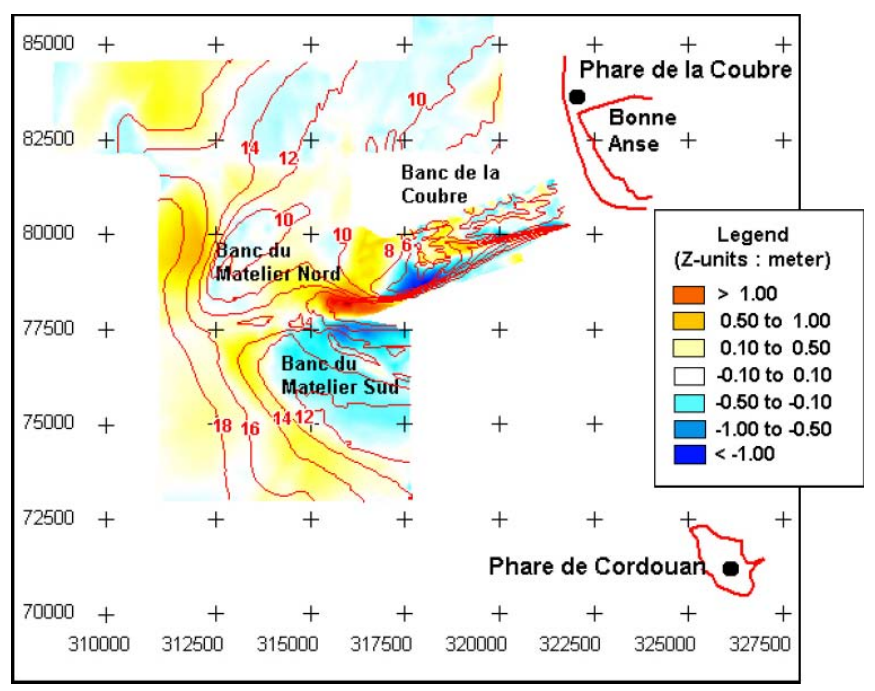

Figure 9 : Evolution morphologique des fonds entre 1994 et 2000 (ramenée à une année) issue des levés bathymétriques du Port Autonome de Bordeaux

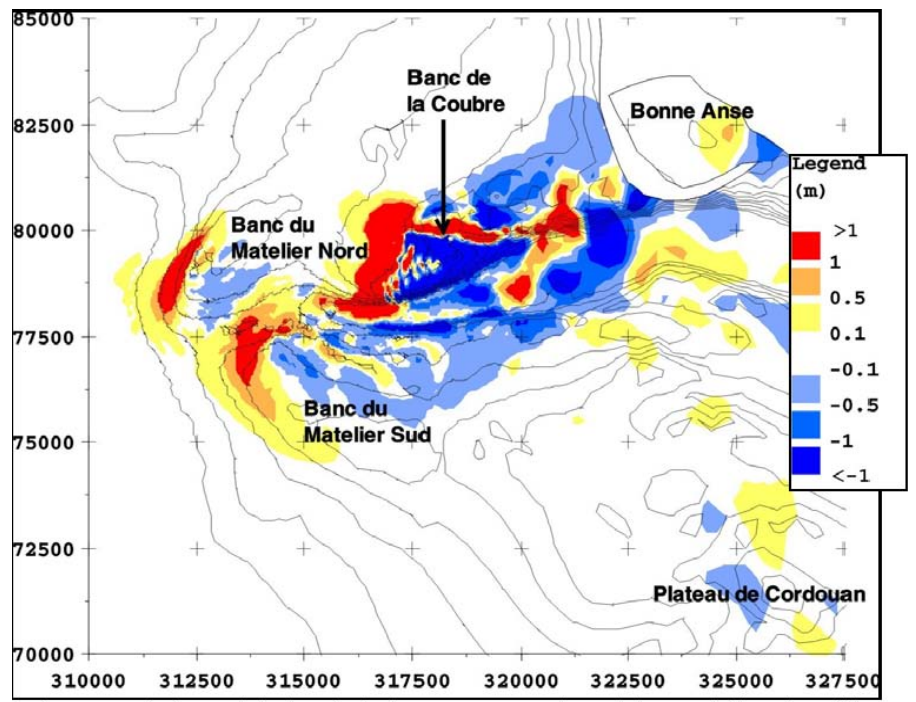

Figure 10: Evolution morphologique des fondsentre 1994 et 2000 (ramenée à une année) issue du modèle 


\section{Les perspectives.}

Outre les objectifs principaux du projet, rappelés dans la section 2, trois perspectives d'utilisations du modèle sont prévues :

•évaluation des évolutions morphodynamiques liées à des phénomènes exceptionnels (crue de la Gironde, tempête météorologique et/ou océanique),

-aide à la gestion des dragages du chenal de navigation,

-impact du prélèvement de sable au niveau des bancs de sable.

Pour détailler le dernier point, dans le cadre du projet SANDPIT, nous envisageons d'araser une partie du banc de la Coubre et d'évaluer l'impact de ce prélèvement de sable sur la houle, les courants, la morphodynamique dans la zone. Nous essaierons également d'estimer l'échelle de temps morphologique pour laquelle le banc est totalement ré alimenté.

\section{Références}

1.Feral A., (2001). La passe d'entrée en Gironde : Evolution et Aménagement, Conférence présentée au $6^{\text {ene }}$ colloque du Conservatoire de l'estuaire de la Gironde, Saint Georges de Didonne.

2.Port Autonome de Bordeaux (Direction de l'Equipement), (1996). Caractéristiques granulométriques des sables dragués sur la passe de l’Ouest, Note technique.

3.Cayocca F., (1996). Modélisation morphodynamique d'une embouchure tidale : Application aux passes d'entrée du Bassin d'Arcachon, Thèse Doct., Université de Bordeaux I.

4.Coulet C., (1999). Influence du Changement de Climat sur l'Erosion des Sédiments Côtiers: Application à l'estuaire de la Gironde, Projet européen CLIMEROD - Stage de DESS « Modélisation et Simulation en Mécanique ", Université Joseph Fourier Grenoble I. 